\title{
Meta-Analysis of Chinese Coal Miners' Mental Illness under SCL Index System
}

\author{
Sun Shanhui ${ }^{1,2, a}$, Li Hong $^{3, b}$ and Li Zhuangzhuang ${ }^{4, c}$ \\ ${ }^{1}$ College of Mathematics and Statistics, Suzhou University, Anhui, China \\ ${ }^{2}$ School of Management, Chinese University of Mining \& Technology \\ ${ }^{3}$ College of Information Engineering, Suzhou University, Anhui, China \\ ${ }^{4}$ College of Mathematics and Statistics, Suzhou University, Anhui, China \\ asshh10304@163.com, ${ }^{b}$ lih65@163.com, ${ }^{c} 897574320 @ q q . c o m$
}

\begin{abstract}
Objective: Under SCL index system, through the comparative study, this paper investigates whether there are significant differences in mental illness tendency between Chinese coal mine workers underground and on the ground. Methods: This paper uses computer to retrieve in Medline, Sciencedirect, Web of Science, the Cochrane Library, Springerlink, CNKI, Wanfang and Weipu database. The retrieval time is from the very beginning of building database to the March of 2015. After the extraction of available data, Stata 10.1 analysis software was applied to heterogeneity test on the research. Then the paper uses the random effects model to calculate and combine odds ratio as well as 95\% confidence interval. Results: 15 articles were finally included by using Meta analysis. Collating data of the experimental group and control group, this paper carries on forest figure mapping, publication bias test, sensitivity analysis and cumulative Meta analysis, and one study result is found to have great deviation impact on the overall conclusion. Eliminating the above research result, the results are greatly improved. Conclusion: Through the result of the Meta analysis, this paper concludes that there are significant differences in mental illness tendency between Chinese coal mine workers underground and on the ground.
\end{abstract}

Keywords: Mine worker; Mental illness; Mental health; Meta-analysis; State

\section{Introduction}

China miners are a huge special occupational group, whose working environment and production activities are with great particularity, so they face much greater survival pressure and pressure of life than other groups. However, due to the neglect of the miners' psychological problems, safety accidents are caused when they work under the complex and even dangerous conditions underground [1]. The safety of coal mine an important task of maintaining social stability and building a harmonious society, and meanwhile the mental health status of the mine workers is closely related to coal mine safety production.

With the development of industrial and agricultural production, as well as change of life style and patterns of behavior, people realize that the coal mine workers' mental health status is closely related to coal mine safety production. Coal miners are an occupational group who face high damage and disease. The occurrence of coal mine accidents are mostly caused by human behavior, which is dominated by psychology. So the miners' psychological factors must be paid attention to when people research on the safety work of coal mine production. According to a new concept of health given by the World Health Organization, health is not only the absence of disease or weakness, but the perfect condition of physical, mental health and social adaptation [1].

It is recognized that many health problems are closely related to people's psychological state, so occupational groups with different work environment and organizations have 
their own mental health problems. Coal production is mostly underground, and underground working conditions are very complex. Water, fire, gas, coal dust, coal mining roof are five natural disasters which seriously threatened the life safety of mine workers. At the same time, occupational hazard factors of coal mine dust, noise, vibration, high temperature, high humidity and poor production organization mode and poor posture, etc, are affecting the physical and mental health of miners in different degrees when they work underground. Psychological research confirms that changes in the objective environment are the most important factor which influences people psychology [2].

Coal miners are occupational groups with high injury rate and high rates of disease. The former research of miner's health focused on the impact of harmful factors such as dust, noise which are caused by production process, on the miners' health. But the research of the impact of the special natural and social environment on the miners' psychological health is not enough [3]. With the development of industrial and agricultural production, as well as change of life style and patterns of behavior, people realize that the coal mine workers' mental health status is closely related to coal mine safety production.

By collecting the research data of the coal mine workers from the symptom check-list 90 (SCL - 90) and general situation questionnaire, from the perspective of system analysis, this paper studies the potential impact of special natural and social environment on the coal mine workers' psychological effects as well as data analysis of investigation statistics on the coal miners suffering from mental illness. SCL-90 is a standardized symptom check list, which can well reflect the change of the mental health status and the severity of mental illness, so it has been widely used in mental health assessment of people. From a technical perspective, this article mainly provides scientific basis for the health education and psychological care of the special group through Meta analysis [4-7]. This article aims to provide basis for the coal mine safety management and maintain the miners' physical and mental health by the study of the mental health status of the coal mine workers and comparative analysis of mental health characteristics of miners. Innovative points include:

First of all, from the perspective of system evaluation, this paper studies whether the Chinese coal mine workers have a tendency to have mental problems. The results of the study can not only summarize and sort out the present situation of Chinese coal mine workers' mental illness and the main problems, but also study the average levels of Chinese coal mine workers' mental status from the overall perspective. By summarizing main problems and average levels of Chinese coal mine workers' mental illness, this paper can provide a basis for psychological counseling and intervention.

Secondly, in the process of concrete analysis of Chinese coal mine workers' mental health, this article uses the method of Meta analysis. During the evaluation and study of the mental health system, many scholars use the Meta analysis techniques. But no scholar has ever been found to use Meta analysis method in the field of research on mental illness of mine workers,

Thirdly, during the study of mental illness tendency of Chinese coal mine workers, according to the Meta analysis technology, the author uses the method of eliminating part of the research results. Studies show that Meta analysis results can be better improved after eliminating part of the research results.

Finally, according to the results of the analysis, combining with the actual situation of China coal production and the humanistic care of mine workers in the process of economic transformation, this article puts forward the relevant countermeasures and suggestions of solving Chinese coal mine workers' mental illness. 


\section{Literature Retrieval and the Choice of Data}

\subsection{Literature Retrieval}

This paper uses computer to retrieve in Medline, Sciencedirect, Web of Science, the Cochrane Library, Springerlink, CNKI, Wanfang and Weipu database. The retrieval time is from the very beginning of building database to the March of 2015. The English retrieval words for this paper are "mine worker, mental health and Meta Analysis". The Chinese retrieval words are "coal miner; mental health". This article filters the data according to the criteria of inclusion and exclusion.

\subsection{The Choice of Data}

In the process of analyzing the mental illness tendency of coal miners by using the SCL index system, most of the authors choose the experimental group and the control group to compare and few of the authors choose the underground group and the ground group to compare. Both have their own research and statistical significance, but essentially, two different choosing ideas are all comparative investigation and statistical analysis of coal miners' mental health problems who work under harsh environments. So, this article studies both of the literature [8]. On the basis of this study, with the methods of the sensitivity analysis and the cumulative analysis, it can be verified that the research idea of this paper is feasible and has a scientific basis.

2.2.1. The Criteria of Inclusion and Exclusion: Inclusion criteria: (1) type of study: This paper includes published randomized controlled trials and non-randomized controlled trials (such as: case-control test, cohort studies, etc.). Only English and Chinese literature can be included; (2) object of study: Through the research based on SCL index system, this paper specializes in the study of mental health problems of coal miners.

Exclusion criteria: (1) this paper uses reviews, lectures, abstracts and review of the literature to exclude. (2) Literature which is unable to access the full text or is published several times, as well as literature which is of non-clinical studies or is of no controlled study will be excluded.

2.2.2. Literature Quality Evaluation: Two researchers are chosen to read the title and abstract of the literature independently. After excluding the tests which clearly does not meet the inclusion criteria, the two researchers study the whole test that might meet the criteria to determine whether they meet or not. And then they exchange to check the tests. If there are differences, the two researchers will discuss to resolve or ask the third researchers to determine whether they can be included.

2.2.3. Literature Retrieval and Data Processing: Try to extract the information of the author, research type, release date, the number of participants, etc. The paper search process is shown in figure 1. 2457 articles were obtained after the first examination. After examining the title and abstract, 2457 articles were excluded. So there are 1321 related articles were firstly included, in which 1123 articles were further excluded, 143 articles were unable to access the full text for various reasons, 35 articles were of no controlled study, 8 articles could not extract data. 12 articles were finally included [9-20]. 


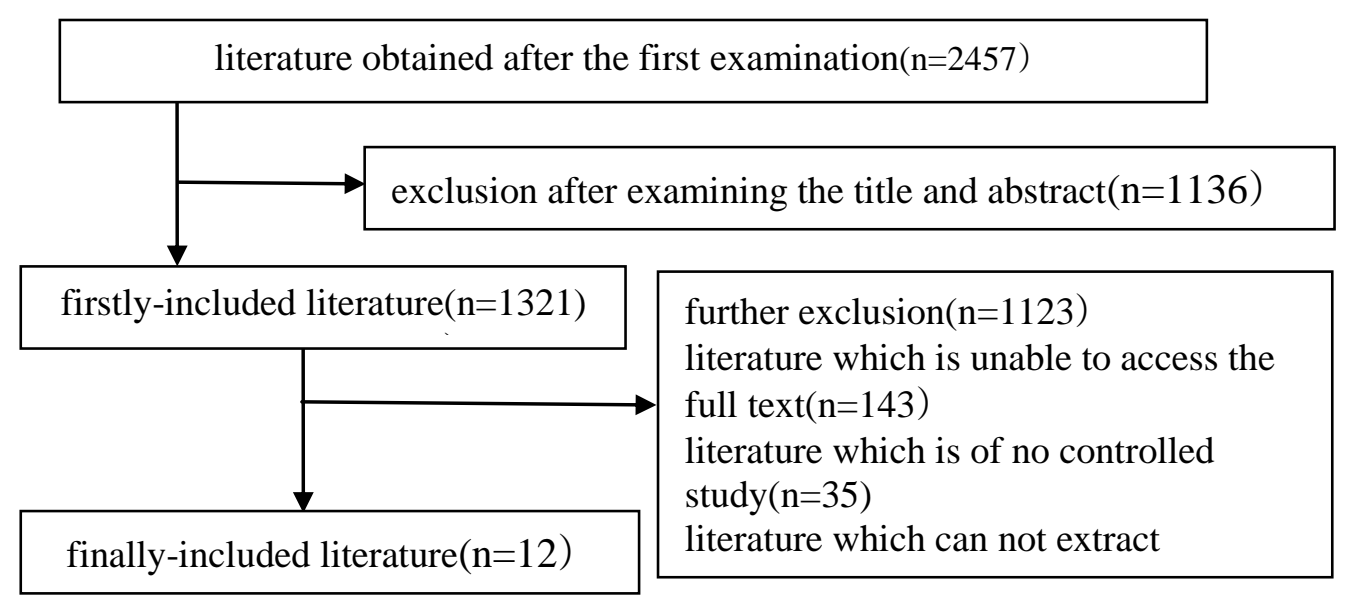

\section{Figure 1. Flow Diagram of Paper Search Process about Chinese Coal Miners' Mental IIIness}

The basic information and related data of the coal mine workers' mental illness tendency in the 12 articles are shown in Table 1.

Table 1. Basic Information of the Included People

\begin{tabular}{|c|c|c|c|c|c|c|c|}
\hline \multirow{2}{*}{ Author } & & \multicolumn{3}{|c|}{$\begin{array}{c}\text { The experimental } \\
\text { group }\end{array}$} & \multicolumn{3}{c|}{ The control group } \\
\cline { 3 - 8 } & Year & n1 & Mean1 & SD1 & n2 & Mean2 & SD2 \\
\hline Shan Changyan & 2011 & 215 & 1.3 & 0.56 & 213 & 1.4 & 0.51 \\
\hline Zhong Minggui & 2012 & 491 & 1.3 & 0.73 & 1388 & 1.29 & 0.42 \\
\hline Chu Jianping & 2011 & 120 & 1.42 & 0.51 & 120 & 1.41 & 0.36 \\
\hline Wang Guishan & 2008 & 100 & 1.53 & 0.42 & 100 & 1.31 & 0.39 \\
\hline Yang Hongxia & 2011 & 1636 & 1.28 & 0.44 & 1388 & 1.29 & 0.42 \\
\hline Dai Fuqiang & 2009 & 14341 & 1.31 & 0.47 & 1388 & 1.29 & 0.42 \\
\hline He Liufang & 1998 & 249 & 1.45 & 0.69 & 724 & 1.32 & 0.44 \\
\hline He Liufang & 1998 & 142 & 1.49 & 0.66 & 107 & 1.4 & 0.73 \\
\hline Wang xianying & 2009 & 1640 & 1.27 & 0.44 & 1388 & 1.29 & 0.42 \\
\hline Song Zhifang & 2010 & 800 & 1.37 & 0.41 & 724 & 1.32 & 0.44 \\
\hline Song Zhifang & 2010 & 400 & 1.56 & 0.56 & 400 & 1.18 & 0.26 \\
\hline Wu Zheng & 2009 & 195 & 1.61 & 0.59 & 1388 & 1.29 & 0.42 \\
\hline Wu Zheng & 2009 & 142 & 1.49 & 0.66 & 107 & 1.4 & 0.73 \\
\hline Li Hongbo & 2013 & 168 & 1.35 & 1.1 & 209 & 1.2 & 0.6 \\
\hline Wang Wenming & 2003 & 264 & 0.42 & 0.13 & 1388 & 1.29 & 0.42 \\
\hline
\end{tabular}

\section{General Introduction of Meta Analysis and Selection of Statistical Methods}

\subsection{General Introduction of Meta Analysis}

The original meaning of Meta analysis is to collect plenty of research results from the literature and summarize them after statistical analysis. Meta analysis means "more comprehensive", marking a more comprehensive integration or extraordinary. In many 
literatures, there are many names for it, such as overview, pooling analysis, quantitative synthesis and so on. But up to now, only Meta-analysis can be used as a dedicated statistical term basically. In 1976 British education psychologist Glass defined it as a research method to give the comprehensive evaluation and quantitative analysis of multiple research results which are mutual independent and have the same purpose. Metaanalysis is essentially a series of process of summarizing multiple research results with the same study purpose and giving analysis and evaluation of the combined effect.

Meta analysis has been more and more widely used in the biological medicine, psychology, education, social science and other fields. In summary it has the following main functions: First, meta-analysis can increase the statistical power and improve the demonstration strength of preliminary conclusions and ability of effect analysis and assessment. Because a single research sample is too small to clearly make sure of some kind of effect. It requires a larger sample to determine and eliminate these effects statistically [21]. So using Meta analysis can merge to analyze the comparable single bed test results, so as to improve the estimate of the effects or effectiveness; second, Meta analysis can make the conclusion more accurate through the analysis of the differences in the results of the study. From the angle of application analysis, Meta analysis can reveal the uncertainties existing in the individual. Then through the method of heterogeneity inspection, it examines the reason of heterogeneity and all kinds of possible bias. Metaanalysis can carry on the contrast research on the effect scale of each research, so it can effectively improve the accuracy and provide the basis for a more scientific and comprehensive for further research and policy-making. Finally, a new hypothesis can be sought. Meta analysis can not only answer the questions which have never been mentioned or can not be solved in individual studies, but comprehensively evaluate multiple results with the same study objective. Comprehensive evaluation results may raise new issues or new hypothesis.

\subsection{Selection of Statistical Methods}

Statistical software uses Stata software, and heterogeneity analysis uses $\chi^{2}$ to test. If inclusion study has clinical homogeneity and $\mathrm{P}>0.05, \mathrm{I}_{2} \leq 50 \%$, statistical heterogeneity is small. So fixed-effects model can be used to analyze the study object. When $\mathrm{P} \leq 0.05$, $\mathrm{I}_{2}>50 \%$, statistical heterogeneity is large. So subgroup analysis is needed to analyze sources of heterogeneity. If there is no exact cause of heterogeneity, random-effects model can be used to analyze. If the study has obvious clinical heterogeneity, only a descriptive research is needed. Weighted mean difference is used in continuity variables with the same measurement units, while standard mean difference is used in continuity variables with the different measurement units [22]. Both of them use 95\% CI. Egger and Begg analysis of STATA12.0 software is used to analyze publication bias with Begg's funnel plot. When $\mathrm{P} \leq 0.1$, it shows that there is a significant statistical bias.

\section{Meta Analysis Results of Coal Mine Workers'Mental Illness Tendency under the SCL Index System}

\subsection{Forest Figure}

Based on the statistical indicators and statistical analysis, forest figure is figure mapped with the results of numerical computing. In plane rectangular coordinate system, it uses a vertical invalid line as the center. Then it uses multiple lines which are parallel to the horizontal axis to describe the influencing quantity and confidence interval of each objects included. And it uses a diamond or other graphic to describe the influencing quantity and confidence interval of each objects included. It simply and visually describes the statistical results of Meta analysis, so it is the most common result expression used in the meta-analysis. 


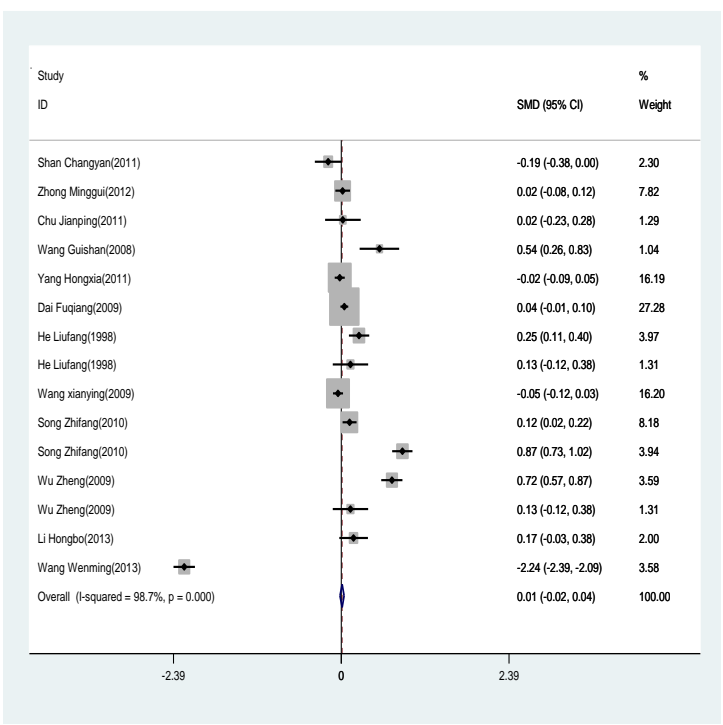

Figure 2. Forest Figure of Chinese Coal Miners' Mental IIIness Under SCL Index System

By mapping the forest figure, the obtained merged SMD value is $0.01,95 \% \mathrm{CI}$ is -0.02 , 0.04 , as shown in Figure 2. Heterogeneity between experimental group and control group is larger $\left(\mathrm{I}_{2}=98.7 \%\right)$. The results of the random effects model suggest $\mathrm{F}_{0}$ has obviously increased, so the difference has no statistical significance.

\subsection{Publication Bias}

Publication bias refers to the research results with statistical significance are easier to contribute and be published than the research results without statistical significance. The researchers may think that the research results without statistical significance are of little significance, so they might not publish or delay publication. Because of publication bias, it is impossible to completely include in all the related research even with careful search strategies and tools. Publication bias may lead to errors of Meta analysis, so it might affect the relevant treatment and decision [23].

The most common system error of the Meta analysis is publication bias, as shown in Figure 3. Through detecting the publication bias of the included literature by using Egger analysis, it shows that $\mathrm{t}=-2.7, \mathrm{P}=0.018<0.05$. Most points are found to be distributed on one side of the tropic from the distribution plot of included literature. From the comprehensive analysis of data and graphics, it can be judged that publication bias may exist in the included literature.

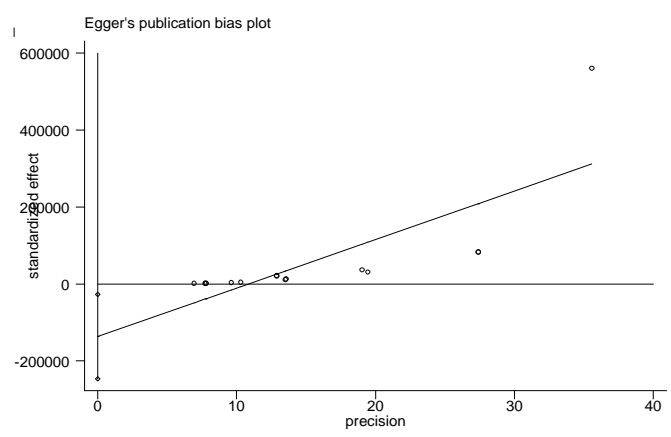

Figure 3. Publication Bias of Chinese Coal Miners' Mental Illness Under SCL Index System 


\subsection{Sensitivity Analysis}

So-called sensitivity analysis is a kind of detection means. It primarily detects the stability of the target object on the assumed conditions and it aims at analyzing all influential factors of Meta analysis. In research activities of sensitivity, all factors that may affect the research activity are eliminated firstly. Then Meta analysis is used to compare the results before and after eliminating the affecting factors.

Sensitivity analysis refers to eliminate all abnormal factors that may affect the research activity. Then Meta analysis is used to compare the results before and after eliminating the affecting factors. The impact of abnormal factors on the consolidation effect will be further analyzed. If there is no fundamental difference in the results of the study by comparing the results, it shows that the Meta research results are credible. Conversely, it shows there are certain key factors that affect the effect in the whole research process, which must be treated with caution, for in-depth analysis of the key factors is good to find the source of them.

The specific process is: Firstly, by summarizing the overall study results of coal mine workers' mental illness tendency, the overall level of their mental illness can be obtained. Secondly, combining the sensitivity analysis, the research results that greatly influence the overall result can be got. Finally, by eliminating the above research results, sensitivity analysis is used again to detect whether the analysis results have obvious improvement. If so, eliminate them. Otherwise, reserve them.

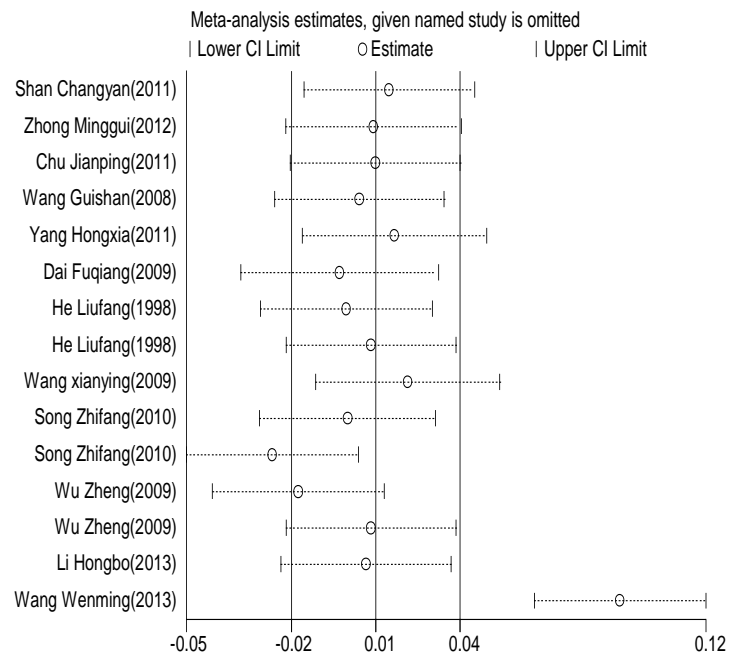

\section{Figure 4. Sensitivity Analysis of Chinese Coal Miners' Mental Illness Under SCL Index System}

As shown in Figure 4, the conclusion of Wang Wenming drew in 2013 was obviously different from other conclusions. His results must be carefully read through and investigated to decide whether delete or not.

\subsection{Cumulative Meta-Analysis}

Cumulative meta-analysis regards a series of tests of the same research purpose as a dynamic, continuous whole. According to a specific order (for example, the publication time, sample size, etc.,), a cumulative meta-analysis is used when adding a research. Therefore, the cumulative Meta analysis is not a new method of statistical analysis, but a method to show a series of research. The essence of the cumulative Meta analysis is a standard meta-analysis. 


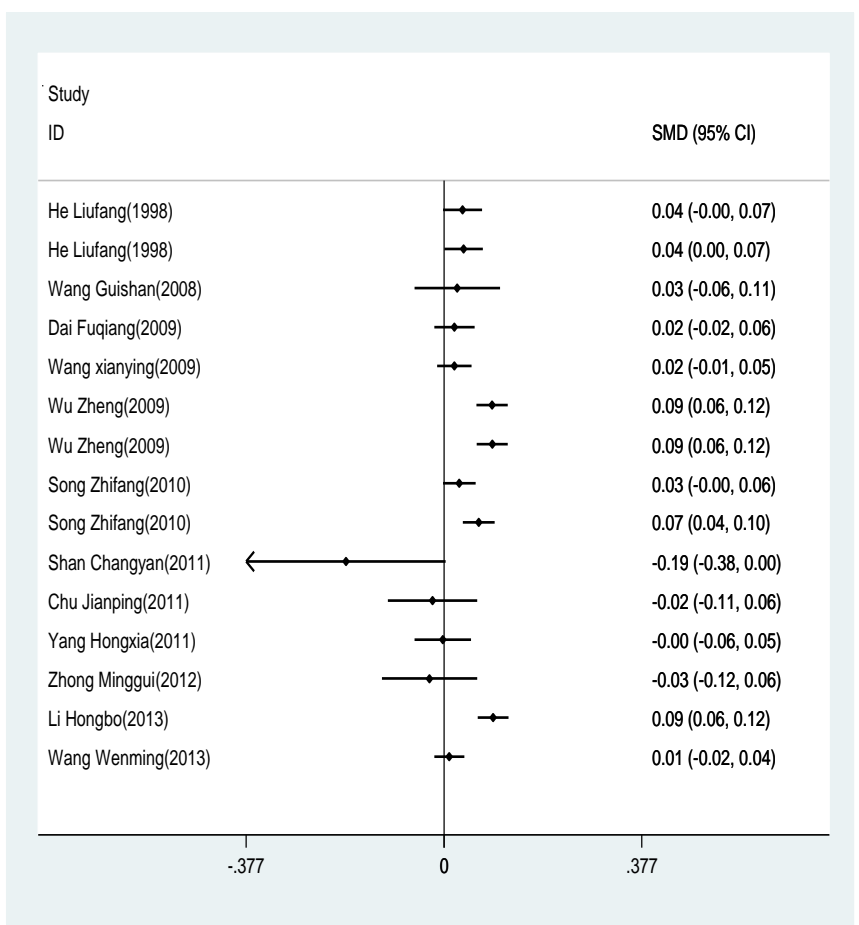

\section{Figure 5. Cumulative Meta Analysis Figure of Coal Mine Workers' Mental Illness Tendency}

Figure 5 shows research results are basically distributed on both sides of a vertical except for one or two results of a great leap according to the order of publication year. The final output result for SMD value is $0.01,95 \%$ CI is $(-0.02,0.04)$, which shows good cumulative effects.

\section{Further Discussion on Meta Analysis Results of Mine Workers' Mental Illness Tendency}

\subsection{Discussions on Excluding the Research Results}

By carefully studying Wang Wenming's papers, according to the introduction of the author in this paper, the mining area chosen to be investigated and studied is a modern mine. Labor intensity of the underground coal mine workers is larger, but the income is higher, so their living conditions and working conditions have been greatly improved. In addition, the author finds that survey and research time of Wang Wenming is 2003, when the benefit of China's coal mining enterprises is generally good. Therefore, the labor intensity of workers on the ground is also large as workers underground, but the income is lower than them. In addition, the person in charge of the mine pays little attention to their working environment. So the ground workers bear greater psychological pressure than underground workers. The reasons for the differences between Wang Wenming's results and other results are found. His research results obtain in a specific period of time and a specific mining area [24]. For the main study matter of this paper, Wang Wenming's results cause obviously particular great impact on this article's research results, so the author decides to delete the research results of his paper so as to objectively and impartially research on the tendency and severity of the coal mine workers' mental illness.

\subsection{Further Interpretation of basic Meta analysis Results}

After excluding the research results of Wang Wenming's paper, the author reprocess the 
new data. From the forest figure, merged SMD value is $0.0094,95 \% \mathrm{CI}$ is $(0.064,0.123)$. Heterogeneity between experimental group and control group is larger $\left(\mathrm{I}_{2}=94.3 \%\right)$. The results of the random effects model suggest the difference has no statistical significance.

Through detecting the publication bias of the new data, the results are shown in Figure 6: Through detecting the publication bias of the included literature by using Egger analysis, it shows that $\mathrm{t}=-1.68, \mathrm{P}=0.118>0.05$. Most points are found to be distributed on two sides of the tropic from the distribution plot of included literature. From the comprehensive analysis of data and graphics, it can be judged that the included literature has no publication bias.

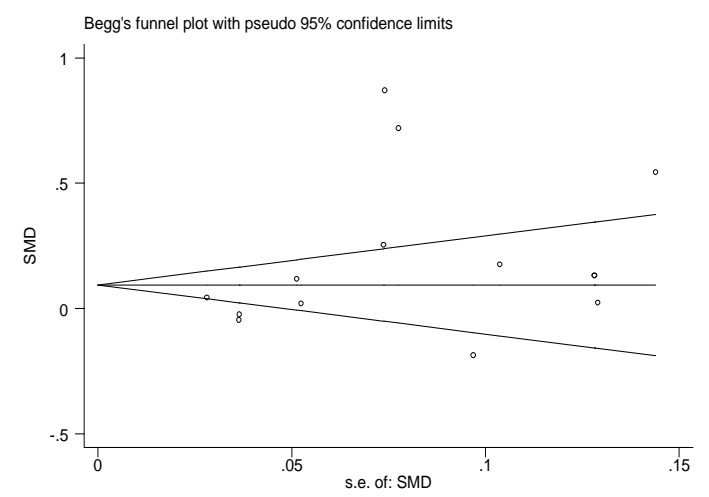

Figure 6. Publication Bias Graphs after Updating Data

In the process of carrying out sensitivity analysis on the new data, through the graphical analysis results, the resulting gaps between the various researches are not big. They are generally distributed in the range, the center of which is 0.94 and $95 \% \mathrm{CI}$ is $(0.064,0.13)$. Through the comparison with Figure 7 , the quality of Meta analysis can be found to be improved greatly after excluding Wang Wenming's research results. The results of sensitivity analysis also directly demonstrate that it is a right decision of excluding Wang Wenming's research results.

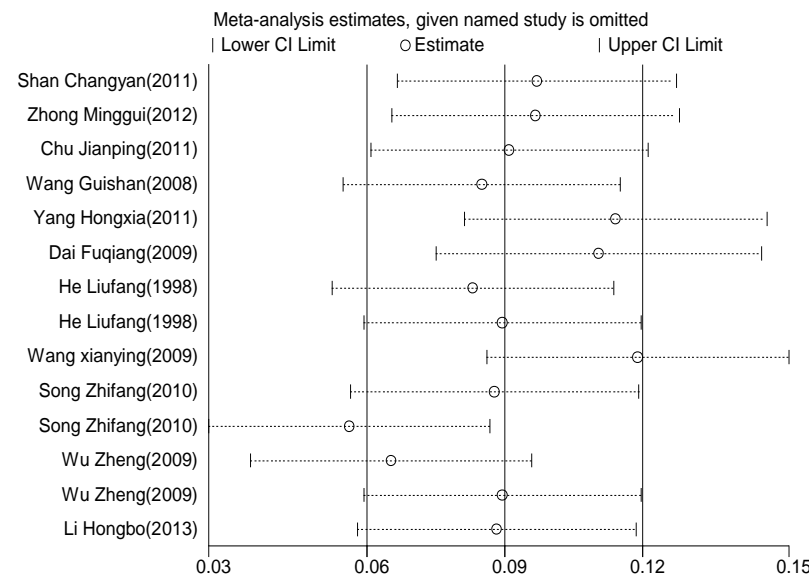

Figure 7. Sensitivity Analysis Graph after Updating Data

Then the cumulative meta-analysis was carried out on the new data, the final output for SMD value is $0.09,95 \% \mathrm{CI}$ is $(0.06,0.06)$, as shown in Figure 8 . The cumulative effect is also very good. By comparing Figure 5 and 8, with the cumulative Meta analysis according to publication years, the results of Figure 8 are more concentrated than the 
results of Figure 5. Therefore, the cumulative results of meta-analysis also show that it is a right decision of excluding Wang Wenming's research results from another side.

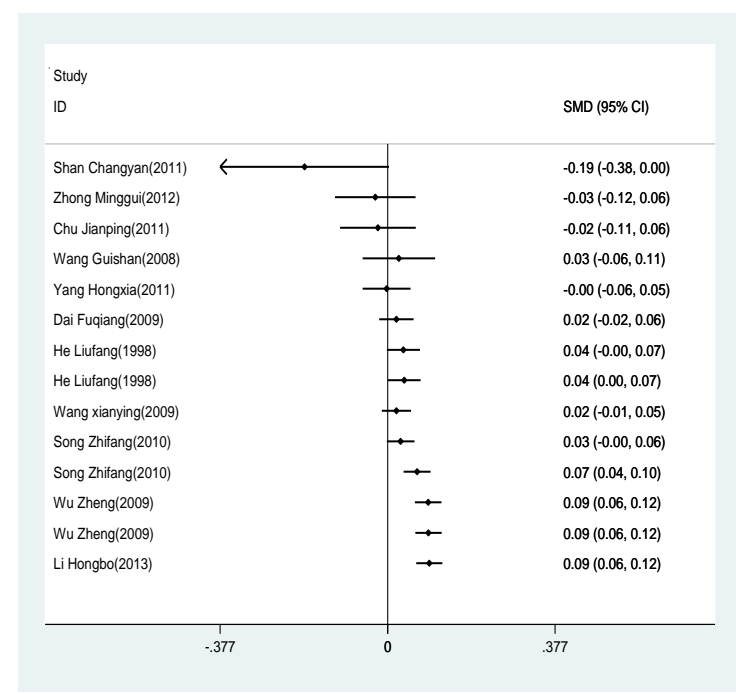

Figure 8. Cumulative Beta-Analysis Graph after Updating Data

\section{Discussion}

6.1 Through the result of the Meta analysis, this paper concludes that there are significant differences in mental illness tendency between Chinese coal mine workers underground and on the ground. Through the results of this survey, the overall mental health level of the coal mine workers underground is lower than the coal miners on the ground or general domestic sample [25]. Mental health problems of the coal mine workers underground are more serious, especially in aspects of body, force, hostility and interpersonal relations. Therefore, the underground operation in coal mine has closely related effects which can not be ignored on coal mine workers' mental health. This may be related to high intensity of long term underground work, high concentration of energy, the joint action of the dust and noise in the workplace and physical fatigue.

6.2 Working conditions of the underground operation in coal mine are poor. There are many complex hazard factors of the occupational disease. The coal mine workers are workers with heavy workload, with high intensity of labor, long working hours, low education level, less hobbies, low pay and social status. In addition, mine disasters sometimes happened and security incidents around them appeared, so they bear much greater psychological pressure.

6.3 According to the common mental health problems of the coal mine workers, the author recommends to carry out mental health education of them. Show them not only the dangers of physical and chemical factors on the human body, the importance of improving working conditions and safety in production, but also how to eliminate unhealthy psychological state, improve interpersonal relationships, as well as promote physical and mental health [26]. Psychological files for the miners who have psychological problems should be established, accompanying psychological guidance, adjustment of the work environment or drug therapy, in addition to the follow-up investigation, so as to eliminate hidden dangers of safety production in coal mine and escort the safety production.

6.4 During the management process of production in coal enterprise, various aspects of the miners' personality characteristics, mental health factors and so on should be taken into consideration. Personality characteristics and post adaptability of miners should be paid attention to. Psychological counseling should be strengthened to improve their psychological quality, so they can regulate and control personal emotional states by 
themselves [27]. Then they can quickly and decisively react to the change of different situation underground in production process. So many accidents will be prevented.

\section{Conclusion}

With the development of the society as well as the progress of science and technology, coal mining technology and equipment is more and more advanced, but the potential dangers of underground work are inevitable. This paper attempts to explore a research study on mental health of coal mine workers, so as to provide a research method and train of thought for people who engage in research on mental health and for people who work for intervention and care of mental illness.

\section{Acknowledgments}

This Work were supported by the Natural Science Foundation of Anhui Provincial Education Department(KJ2012A263), the Scientific Research Platform Open Topic of Laboratory of Intelligent Information Processing (2014YKF39), the Open Project of Collaborative Innovation Center of Suzhou Regional Development(2014SZXTKF06), the Excellent Academic and Technical Backbone of Suzhou University(2014XJGG03).

\section{References}

[1] R. Phillip, L. Shi, G. L. Na and L. Z. Yuan, "Stress and health”, China light industry press, Beijing, (2000).

[2] P. S. Paul, "Predictors of work injury in underground mines-an application of a logistic regression model”, Mining Science and Technology (China), vol. 19, (2009) May, pp. 282-289.

[3] K. A. Tawiah, S. Leka, A. Jain, D. Hollis and T. Cox, "The impact of physical and psychosocial risks on employee well-being and quality of life: The case of the mining industry in Ghana", Safety Science, vol. 65, (2014) June, pp. 28-35.

[4] L. K. Chapman, J. Petrie and L. Vines, "Factor structure analysis of the SCL-90-R in a communitybased sample of African American women", Psychiatry Research, vol. 199, (2012) September, pp. 140144.

[5] J. Zhang and X. Zhang, "Chinese college students' SCL-90 scores and their relations to the college performance", Asian Journal of Psychiatry, vol. 6, (2013) April, pp. 134-140.

[6] P. Bech, J. Bille, S. B. Møller, L. C. Hellström and S. D. Østergaard, "Psychometric validation of the Hopkins Symptom Checklist (SCL-90) subscales for depression, anxiety, and interpersonal sensitivity", Journal of Affective Disorders, vol. 160, (2014) May, pp. 98-103.

[7] D. Helm and D. Eis, "Subgrouping outpatients of an environmental medicine unit using SCL-90-R and cluster analysis", International Journal of Hygiene and Environmental Health, vol. 210, (2007) December, pp. 701-713.

[8] T. C. Davies and H. R. Mundalamo, "Environmental health impacts of dispersed mineralization in South Africa", Journal of African Earth Sciences, vol. 58, (2010) November, pp. 652-666.

[9] S. Changyan and W. Junguang, "RPN promote the coal mine enterprise staff mental health research", Theory Research, vol. 17, (2010) November, pp. 94-95.

[10] Z. Minggui, L. Lei and L. Feifei, "Survey and Analysis on Mental Health Status of Coal Mine Workers and Groud Mine Workers", Journal of Anhui Vocational and Technical collage, vol. 11, (2012) March, pp. 100-101.

[11] C. Jianping, C. Yunsheng and C. Xiangdong, "Study on Personality Traints, Mental Health and Work Injury of Coal Miners", China Journal of Health Psychology, vol. 19, (2011) June, pp. 664-665.

[12] W. Guishan and C. Yiren, "A Study of Mental Health and the Work Injury of Coal Miners", Journal of Psychiatry, vol. 21, (2008) May.

[13] Y. Hongxia, W. Xianying, S. Jinxia and W. Qinglian, "Investigation on Relationships among Social Support, Life Events and Phschological Health of Coal Mine Workers", Chinese Nursing Research, vol. 25, (2011) February, pp. 495-497.

[14] D. Fuqiang, Z. Yonghua, J. Xiulan, W. Lingyun and D. Hao, "Mine workers mental health investigation and analysis", Journal of Qiqihar Medical College, vol. 30, (2009) August, pp. 1929-1930.

[15] H. Liufang and Y. Fang, "The psychological health level of coal miners", Chinese Rural Health Service Administration, vol. 18, (1998) September, pp. 55-56.

[16] W. Xianying, Y. Hongxia, L. Jianguo, L. Min, L. Jingya and Y. Xue, 'Influence of Coal Miners' Mental Health Guidance on Production Safety of Coal Mine", Chinese Nursing Research, vol. 23, (2009) November, pp. 3050-3051. 
[17] S. Zhifang, L. Dezhi, Z. Huijun, J. Xiaomin and Z. Meirong, "Study on Mental Health Level of Coal Mine Workers", China Journal of Health Psychology, vol. 18, (2010) January, pp. 48-50.

[18] W. Zhen, W. Qiong and L. Jie, "Investigation on the Status and Related Factors of the Mental Health of Coal Miners Who Worked Underground", China Journal of Health Psychology, vol. 17, (2009) December, pp. 1508-1510.

[19] L. Hongbo, H. Xue, Z. Yun and Y. Zhen, "Comparative Study on Post-Traumatic Mental Health Status among Coal Miners", Chinese Journal of Coal Industry Medicine, vol. 16, (2013) October, pp. 17331735 .

[20] W. Wenming, D. Bo, M. Li and Z. Yin, "Mental health survey underground workers", Chinese Journal of Clinical Rehabilitation, vol. 7, (2003) February, pp. 488-488.

[21] H. Chen, Q. Feng, R. Long and H. Qi, "Focusing on coal miners' occupational disease issues: A comparative analysis between China and the United States", Safety Science, vol. 51, (2013) January, pp. 217-222.

[22] J. A. Naslund, K. A. Aschbrenner, L. A. Marsch, G. J. McHugo and S. J. Bartels, "Crowdsourcing for conducting randomized trials of internet delivered interventions in people with serious mental illness: A systematic review", Contemporary Clinical Trials, vol. 44, (2015) September, pp. 77-88.

[23] B. Happell, R.Stanton, W. Hoey and D. Scott, "Knowing is not doing: The relationship between health behaviour knowledge and actual health behaviours in people with serious mental illness", Mental Health and Physical Activity, vol. 7, (2014) September, pp. 198-204.

[24] H. H. Wang, Z. J. Zhang, Q. R. Tan, H. Yin, Y. C. Chen, H. N. Wang, R. G. Zhang, Z. Z. Wang, L. Guo, L. H. Tang and L. J. Li, "Psychopathological, biological, and neuroimaging characterization of posttraumatic stress disorder in survivors of a severe coalmining disaster in China", Journal of Psychiatric Research, vol. 44, (2010) April, pp. 385-392.

[25] K. N. Carlisle and A. W. Parker, "Psychological Distress and Pain Reporting in Australian Coal Miners", Safety and Health at Work, vol. 5, (2014) December, pp. 203-209.

[26] K. Anargul and M. Maria, "Subjective Study of Risk Assessment in Miners Work", Procedia - Social and Behavioral Sciences, vol. 82, (2013) July, pp. 908-912.

[27] L. Hongxia, F. Yongbin, T. Shuicheng, L. Fen and L. Huan, "Study on the Job Stress of Miners", Procedia Engineering, vol. 84, (2014) September, pp. 239-246.

\section{Author}

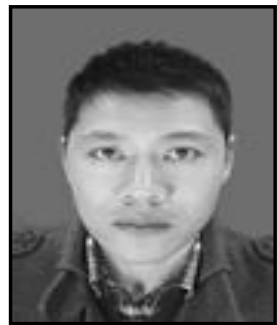

Sun Shanhui, he is now a doctoral candidate in Management science and engineering in China University of Mining \& Technology, China. He is currently an associate professor in the college of mathematics and statistics at Suzhou University. His research interests include fractal geometry and management science and engineering. 